\title{
The Role of Leptin in Obesity-Induced Hypertension
}

\author{
Article by Sarah Miraaj-Raza, Erika J. Pearce, Chuku Okorie, Amitabha Ray* \\ *Faculty of Basic Sciences, Saint James School of Medicine, Anguilla, British West \\ Indies \\ E-mail: ray.amit213@gmail.com
}

\begin{abstract}
Obesity is linked with the growth of white adipose tissue and associated chronic hyperleptinemia. Leptin is a hormone-like cytokine or adipokine secreted mainly from adipose tissue. High leptin state in obesity rapidly causes selective resistance, focused in the arcuate nucleus of the hypothalamus, and centered round leptin's role in food intake and satiety. This resistance lowers the body's reaction to food intake and prevents the anorexigenic effects of leptin. As this resistance builds up, the intake of food increases causing an enhancement in body adiposity and leptin levels. However, some pathways do not build resistance to leptin and continue to exhibit the stimulatory effects, which cause a persistent stimulation of sympathetic nervous system (SNS), particularly in the kidneys and skeletal muscles. The increase in SNS activity in the kidney, along with the endothelial dysfunction and oxidative stress, lead to an increase in blood pressure. Apart from leptin's effects on SNS and renal function, this adipokine influences vascular health and hypertension through several phenomena or mechanisms such as baroreflex sensitivity, release of nitric oxide and cardiac hormones. In this review, an attempt has been made to highlight different aspects of leptin biology, which are relevant to hypertension.
\end{abstract}

Keywords: obesity, leptin, hypertension, melanocortin, autonomic nervous system

\section{Introduction}

Since the early 1990s, leptin has been a source of much interest in the field of obesity and obesity-related comorbidities. Nevertheless, there remains a great deal we do not understand about how leptin works precisely. It has long been established that obesity increases the risk of metabolic syndrome and cardiovascular disease and that this increased risk is due, at least in part, to the increase in blood pressure that tends to follow increased adiposity. However, discovering the cause of hypertension in obesity could revolutionize the way that we treat obesity-related hypertension. Recent studies have implicated leptin in the pathogenesis of hypertension in the obese population due to leptin resistance in the arcuate nucleus and selective activation of a sympathetic nervous response in the kidneys and skeletal muscle.

In this review, an attempt has been made to focus primarily on different biological mechanisms that are linked to blood pressure regulating role of leptin. For this purpose, PubMed system has been used largely to search for relevant literature. The discussion of this paper (i.e., Critical Review section) has been divided into 13 subtopics. In the first part of this review article, topics like leptin's pathophysiological effects and intracellular signaling have been mentioned. Finally, various important issues such as effects on autonomic nervous system, kidney and cardiovascular systems have been discussed in relation to both health and disease conditions. 
South American Journal of Medicine

Special Edition 2016

\section{Physiological effects of leptin}

Leptin is one of the many adipokines, which plays an important role in maintaining the balance between food intake and energy expenditure, by way of an anorectic role. Leptin is an adipose-derived hormone and is secreted primarily by adipocytes of white adipose tissue (WAT) (Ray \& Cleary 2010). In a physiological state, when energy supply is adequate, leptin is released from adipocytes to alert the hypothalamus, and, in particular, the arcuate nucleus, that enough food has been consumed. Moreover, leptin promotes energy expenditure in the form of thermogenesis and sympathetic nerve activity (SNA) in the kidneys and adrenal glands. When food supply is scarce, leptin levels fall, triggering an adaptive neuroendocrine response. This response leads to decrease in reproductive hormones, thyroid hormone and insulin-like growth factor, and increase in growth hormone to mobilize energy stores (Mantzoros et al. 2011). Leptin also plays a role in wound healing, hematopoiesis, osteogenesis, insulin secretion and sensitivity, and glucose homeostasis (Figure 1).

\section{Pathological effects of leptin in obesity}

In the obese individual, the increase in adiposity creates an increase in leptin secretion and a state of chronic hyperleptinemia. In this state, the role of leptin becomes pathological. The arcuate nucleus becomes selectively resistant to leptin's inhibitory effect on its orexigenic neurons, while continuing to stimulate the neuronal systems in charge of energy expenditure. Leptin continues to act on other hypothalamic targets, which continue to stimulate renal and muscular sympathetic nervous system (SNS) activity (Reed et al. 2010). The SNS stimulation appears to be selective in its targets, generating functional disruption in the autonomic nervous system (ANS). Since the feedback loop between increased leptin levels and satiety has been interrupted, the leptin levels continue to rise, leading to continuous stimulation of sympathetic activity and this imbalance leads to endothelial damage and increased arterial pressure. The mechanism of SNS activation in the state of obesity involves hyperleptinemia, activation of the central nervous system (CNS) melanocortin and renin-angiotensin-aldosterone systems (RAAS), hypoadiponectinemia, hypoghrelinemia, hyperinsulinemia, and baroreflex dysfunction (da Silva et al. 2009; Hall et al. 2015). The primary emphasis of this paper is the impact of the chronic exposure to obesity-induced hyperleptinemia and the cascade of events that ensue.

\section{Leptin and melanocortin system}

While chronic high levels of leptin, which is seen in obesity, have been shown to build increasing resistance to its anorexigenic effects, the stimulation of sympathetic nerve continues to rise as leptin levels rise. To understand how some leptin functions are quelled in chronic hyperleptinemic states while others continue to be stimulated is essential to our understanding of the role of leptin in metabolic dysfunction, hypertension and cardiovascular diseases. Biochemically, leptin simultaneously activates the catabolic pathway of proopiomelanocortin (POMC)/cocaine-and-amphetamine related transcript (CART) neuron and inhibits the anabolic neuropeptide Y/agouti-related protein NPY/AgRP pathway in the arcuate nucleus. The POMC is then processed into melanocortins, such as alpha melanocortin stimulating hormone $(\alpha-\mathrm{MSH})$, in second order neurons in a variety of other hypothalamic nuclei. When leptin binds to the leptin receptors $(\mathrm{Ob}-\mathrm{R}$, long $(\mathrm{Ob}-\mathrm{Rb})$ and short $(\mathrm{Ob}-\mathrm{Ra})$ isoforms) on POMC neurons $\alpha-\mathrm{MSH}$ is released and binds to melanocortin receptors (MCR) throughout the CNS, mostly MC3R and MC4R type receptors (Simonds \& Cowley 2013). Stimulation of MC4R increases 
sympathetic nerve activity to the kidneys and in brown adipose tissue. However, when MC4R receptors are blocked only sympathetic nerve activity to the kidneys is affected (Haynes 2005). Interestingly, MC4R deficient mice are hyperphagic, obese, and display signs of metabolic syndrome, but are not hypertensive, despite high levels of leptin. Likewise, deletion of POMC neuron leptin receptors continue to cause mild obesity, without the hypertensive effects of chronic hyperleptinemia (da Silva et al. 2013). In contrast, yellow Agouti-overexpressing mice are resistant to the anorexigenic effects of leptin while renal SNA remains intact, causing hypertension. These mice are very similar to the diet-induced obesity (DIO) mouse model, indicating that leptin resistance in the AgRP neurons of the arcuate nucleus plays a large role in obesity-induced hypertension (Haynes 2005).

A recent study by Purkayastha et al. (2011) was able to uncouple obesity and

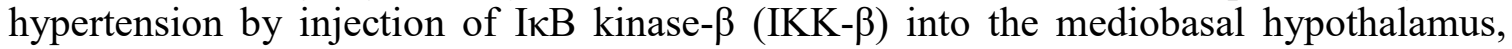
near the arcuate nucleus, and further define the role of POMC and melanocortin system in obesity-induced hypertension, while pointing to AgRP role in hyperphagia. Activation of the pro-inflammatory protein nuclear factor $\kappa \mathrm{B}(\mathrm{NF}-\kappa \mathrm{B})$ and its upstream activator IKK- $\beta$ in the mediobasal hypothalamus rapidly elevated blood pressure in mice independently of obesity. The study also reported that this form of hypothalamic inflammation-induced hypertension involved the sympathetic upregulation of hemodynamics and POMC neurons that has a crucial role in obesity-related hypertension (Purkayastha et al. 2011). In vivo, the stimulation of the IKK- $\beta / \mathrm{NF}-\kappa \mathrm{B}$ pathway has been demonstrated to be activated when leptin binds to $\mathrm{Ob}-\mathrm{R}$ in the arcuate nucleus (Humphreys 2011). These experiments point to the POMC neurons and the downstream effects of $\alpha-\mathrm{MSH}$ on MC4R receptors as the target points for blood pressure regulation by leptin. IKK- $\beta$ was similarly used in experiments by Zhang et al. (2008) to show the role of NPY/AgRP neurons in the homeostasis of body weight. The role of IKK- $\beta / N F-\kappa B$ activation in NPY/AgRP neurons has also been implicated in leptin resistance (Figure 2). These studies showed AgRP/IKK- $\beta$ knockout mice fed a high fat diet were lower in body weight and had preserved leptin signaling, compared to the control group.

\section{Leptin and intracellular signaling}

Though different studies have been able to draw a strong correlation between various neurons of the hypothalamus and their pathophysiological response to hyperleptinemia, the underlying intracellular signaling pathways are not well understood. Selective leptin resistance occurs due to the different biochemical pathways initiated by leptin, and there are a number of negative feedback loop initiators that have been implicated in this complicated process. Studies have shown that even inhibitors of leptin signaling pathways react differently and are preferentially upregulated in different cell types. The model of leptin signaling includes induction of the Janus kinase (JAK) 2/signal transducer and activator of transcription 3 (STAT3) pathway (Frühbeck 2006). When leptin binds with its receptor on the cell surface of a hypothalamic neuron, it initiates a conformational change that leads to intracellular activation of JAK2 and phosphorylation of the Src homology2 domain of STAT3. The phosphorylated STAT3 then dimerizes, travels to the nucleus and regulates gene expression by binding to promotor regions (Kalil \& Haynes 2012). This pathway induces the production of suppressor of cytokine signaling (SOCS) 3 as a negative feedback mechanism. SOCS3 binds to the tyrosine residue (Tyr985), which prevents the phosphorylation of STAT3 (Yang \& Barouch 2007). In high leptin states, these leptin-stimulated pathways are increasingly inhibited by SOCS3 (Figure 3). 
Transgenic mice bred to overexpress SOCS3 in POMC neurons impaired STAT3 signaling, leading to obesity and leptin resistance. However, when the mice were transgenically modified to overexpress SOCS3 in Ob-Rb neurons no obesity was noted and, in fact, the mice were shown to have a small but significant drop in weight and food intake and in leptin levels, compared to their wild type counterparts. This was an unexpected finding and supports the theory that SOCS3 may influence different $\mathrm{Ob}-\mathrm{Rb}$ expressing neurons in different ways (Reed et al. 2010). This was further supported by Pedroso et al. (2014) in their experiments with ablation of the Socs3 gene in various mouse models. They found that when SOCS3 was deleted at the neuronal level, the result was improved glucose homeostasis and partial prevention of DIO. However, when SOCS3 was inactivated only in Ob-R-expressing cells there was no change to the effects of DIO, but these mice were protected against diet-induced insulin resistance. These experiments show the down-regulation of the JAK2/STAT3 pathway by SOCS3 overexpression as would be seen in the obese, hyperleptinemic state. Most significantly, however, AgRP neurons build up resistance to leptin through overexpression of SOCS3 at a faster rate than both POMC neurons and other Ob-R-expressing neurons in other hypothalamic nuclei (Olofsson et al. 2012). This ties in to the clinical picture of the yellow Agouti-overexpressing mice which are resistant to leptin's influence on food intake, but continue to exhibit high levels of SNS activity (Haynes 2005).

Binding of leptin to the $\mathrm{Ob}-\mathrm{Rb}$ receptor also activates mitogen-activated protein kinase (MAPK) pathway through SHP2 and the phosphoinositol 3 kinase (PI3K) pathway via insulin receptor substrate type 2 (Kalil \& Haynes 2012). Though both SHP2 and IRS2 have been implicated in leptin induced intracellular activation, deletion of either of these substances in the entire brain or forebrain, only produce mild hyperphagia and obesity (da Silva et al. 2013). Protein-tyrosine phosphatase-1B (PTP1B) has also been shown to dephosphorylate JAK2 in hyperleptinemia and knockout mice have increased leptin sensitivity and reduced body weight (Mantzoros et al. 2011).

\section{Leptin and blood brain barrier}

A major component of leptin resistance is possibly an impaired transport of leptin across the blood-brain barrier (BBB). Investigators have suggested the existence of an active transport system via Ob-Ra (Rivest 2002). The impaired transport across the BBB could be due to saturation in the transport of leptin and a subsequent decrease in transport activity. This appears to be supported by the decrease in cerebral spinal fluid (CSF) leptin levels to serum leptin levels in obese individuals. However, it has also been noted that obese individuals still have higher CSF levels of leptin than their lean counterparts (Haynes 2005). In addition, hypertriglyceridemia has been demonstrated to inhibit the transport of leptin across the BBB, thus attenuating the leptin signal across the BBB and providing a mechanism for peripheral leptin resistance (Banks 2008). It has also been noted that different brain regions are saturated at different concentrations. When mice models were centrally injected with leptin equivalent to normal limit concentrations for lean humans, the hypothalamus was shown to reveal a preferentially higher concentration than any other brain region. However, when mice were centrally injected with leptin levels mimicking those of obese humans, the hypothalamus showed the lowest leptin concentration of all brain regions. This study implies that different brain regions may have different leptin level thresholds to be activated and may contribute to the clinical finding of selective leptin resistance (Mantzoros et al. 2011). 


\section{Leptin and endothelial function}

Leptin has two antithetical effects that result in a balanced action in a normal physiological state. These actions are simultaneous pressor and depressor effects by inducing activation of SNS causing vasoconstriction and also increase production of nitrous oxide (NO) by the endothelium, mediator of vasodilation. Studies were performed to delineate the role of leptin on endothelium-dependent vasodilation as well as endothelium-independent vasodilation (Freeman et al. 2014). Mice treated with leptin depicted no significant change in endothelium-independent vasodilation in response to sodium nitroprusside. Yet, in response to acetylcholine there was a significant reduction in endothelial-dependent vasodilation in leptin-treated mice. On the other hand, leptin deficient obese mice expressed impaired endothelial relaxation in the presence of acetylcholine that was corrected by exogenous leptin treatment. These experiments have demonstrated leptin as a potent vasodilator and indicated the presence of leptin receptors on endothelial cells (Wang et al. 2013).

Endothelial dysfunction is a result of the oxidative damage that is caused by prolonged state of obesity-induced hyperleptinemic effect of increasing reactive oxygen species (ROS). It is of significance to note that short-term exposure of endothelial cells to leptin has been demonstrated to serve as a benefit by stimulating endothelial NO synthase (eNOS). However, long-term exposure of the endothelial cells to leptin actually resulted in decrease NO availability. Among free radicals, superoxide and peroxynitrite in particular have been shown to be increased through the stimulation of endothelial cells by excessive leptin (Korda et al. 2008). Then, ROS have been recorded to inactivate NO or eNOS and act as a potential cause of endothelial damage (Mattu \& Randeva 2013). When a superoxide scavenger, membrane-permeable piperidine nitroxide tempol, was administered to mice there was no endothelial dysfunction observed under the influence of leptin (Wang et al. 2013). In DIO mouse models, increased leukocyte-endothelial interactions were seen to correspond with the damage of endothelial cells. The functional vascular impairments are predictive of cardiovascular complications that may occur later.

\section{Nitric oxide release}

The role of $\mathrm{NO}$, in a normal physiological state, consists of far more than vasorelaxation. NO has an inhibitory action on oxidation of low-density lipoprotein (LDL), leukocyte migration to the subendothelial space, smooth muscle cell (SMC) proliferation and migration, platelet adhesion and aggregation. NO reduces the expression of adhesion molecules and increases blood flow to hinder coagulation - all function to protect vascular integrity. Studies have shown leptin to participate in phosphorylation of eNOS that leads to NO release. This mechanism was further examined by the administration of leptin to mice that were pretreated with a NOS inhibitor, resulting in an increased blood pressure (Beltowski 2012). Essentially, in non-obese individuals, in the presence of eNOS leptin induces NO release to cause vasodilation, whereas independent of eNOS or in the event of its inhibition, leptin causes vasoconstriction and increase in blood pressure. Hence, it is suggestive that vascular leptin-resistance may exist in obese individuals. In one particular study, leptin was administered in a pulsatile manner to avoid inducing leptin resistance, nonetheless assuring that the same obese levels of leptin were infused. After one week of leptin treatment in this manner, diminished ability of the endothelium relaxation was observed (Wang et al. 2013). Experiments have exhibited impairment of the stimulatory effect of leptin on NO in chronic hyperleptinemia (Beltowski 2012). Therefore, this impairment of leptin induced NO-mediated vasorelaxation probably contributes to leptin-induced hypertension in obesity. 
South American Journal of Medicine

Special Edition 2016

\section{Leptin and SNS activity}

There are two pivotal elements that cause an increase in SNS activity: hyperleptinemia and activation of the brain POMC neurons and MC4R. In obese microenvironment there is selective increase in SNS activity to particular organs. Skeletal muscle and kidneys demonstrate elevated SNS activity whereas due to baroreflex inhibitory effect, cardiac sympathetic activity is minimally or not increased. Leptin activates the SNS by local peripheral actions and by the effects on hypothalamus that are centrally mediated. Studies have shown that acutely administered leptin did not affect the blood pressure if it was injected peripherally, whereas central infusion in DIO rats caused resistance to the actions of leptin in peripheral organs such as the kidney (Freeman et al. 2014).

Studies displayed that within a week of exposure to high fat diets in DIO mice, a rise in SNS activity occurred. Furthermore, the same rise was observed in non-obese mice that were subjected to modest weight gain (da Silva et al. 2013). In animals in which SNS was inhibited, leptin was shown to reduce the blood pressure by other factors including NO-mimetic effect on the endothelium (Beltowski 2012). Again, these studies confirm the normal contrasting function of leptin that involves the balanced activation of vasoconstriction by SNS and vasorelaxation by NO, cancelling out any changes in blood pressure. The only way that the depressor response to leptin can be preserved is by sympathectomy. The factors that contribute to increase SNS activity include blunted baroreflex sensitivity, Angiotensin II release, hyperleptinemia, hypoadiponectemia, hyperinsulinemia, and hypoghrelinemia.

Leptin-induced increase in SNS does not cause hypertension in the absence of angiotensin II. Leptin works in synergy by enhancing the presser effect of angiotensin II in acute or chronic treatment. Rats on high sodium diets have chronic vasoconstriction response to angiotensin II, an effect that is eliminated in the event of celiac ganglionectomy. Consequence of the denervation was recovery of the leptin-induced impairment of endothelial-dependent vasodilation in response to acetylcholine; blockage of leptin-induced supplementation of angiotensin II action to increase systolic blood pressure (Wang et al. 2013).

\section{Leptin and renal function}

Short-term administration of leptin depicted a natriuretic property of the hormone in rats, increasing excretion of sodium and water, primarily acting at the tubular level. However, in the setting of obesity, leptin has not been shown to increase sodium excretion that might cause an abnormal renal-pressure natriuresis. Impairment of this normal sodium excretion function of leptin in hyperleptinemia participates in causing obesity-related hypertension as a result of sodium retention and a rightward shift in the pressure-natriuresis curve (da Silva et al. 2013). It may therefore be suggested that the environment of hyperleptinemia may cause renal leptin resistance similar to central resistance to leptin impairing the anorectic effect of leptin. When obese rats were placed on calorie-restricted diets, renal regeneration of NO took place that then participated in the restoration of the natriuretic function of leptin (Freeman et al. 2014). Potential causes of renal-leptin resistance in obesity-associated hypertension include oxidative stress as a result of excessive degradation of $\mathrm{NO}, \mathrm{Ob}-\mathrm{R}$ downregulation, post-receptor signaling alterations or antinatriuresis resulting from increased activation of the efferent renal SNS. The latter was supported by a study in which kidney nerve supply was surgically removed resulting in the restoration of leptins natriuretic actions. On the other hand, obstruction of endogenous release of leptin reduced excretion of sodium and water by approximately $20-25 \%$ (Freeman et al. 2014). 


\section{Leptin and cardiovascular diseases}

Obesity is a low-grade pro-inflammatory chronic state in which inflammatory cytokines have multifactorial effects causing pathologies preceding cardiovascular diseases (CVD). The prolonged inflammatory state sustained by abnormal production of adipokines constitutes a central role in the formation and progression of atherosclerosis, which is concomitantly present with endothelial dysfunction (Mattu \& Randeva 2013). Leptin also stimulates to express pro-atherogenic and pro-angiogenic factors. Arterial pressure and heart rate are both increased in chronic leptin exposure in mice (Haynes 2005). Along with the heart rate, chronic IV infusion of leptin also increases the mean arterial blood pressure.

\section{Atherosclerosis/Endothelial dysfunction}

There is leukocytic and macrophage involvement in the early developmental stage of atherosclerosis in the endothelial wall. In obesity, adipose tissue consists of an increase in macrophage to adipocyte ratio along with alteration in the phenotype of macrophage. This increase in the macrophage recruitment is in association with systemic inflammation and insulin resistance, both of which are determined by which macrophage subtype is expressed in obesity. There are two main types of macrophages, M1 and M2. M1 is expressed in the adipose tissue of obese individuals, causes release of pro-inflammatory cytokines such a tumor necrosis factor alpha (TNF $\alpha$ ) and interleukin-6 (IL-6), and also promotes insulin resistance. These pro-inflammatory cytokines are involved in the pathogenesis of endothelial dysfunction eventually leading to atherosclerosis. M2 is expressed in the adipose tissue of lean individuals, and involved in secretion of antiinflammatory cytokines and protects against insulin resistance (Nakamura et al. 2014). TNF $\alpha$ is one of the major pro-inflammatory cytokines, predominantly produced by monocytes or macrophages in chronic inflammatory diseases. TNF $\alpha$ induces expression of certain adhesion molecules, which participate in coagulation processes, contributing endothelial dysfunction and preceding atherosclerosis. However, decrease in weight in obesity results in decrease of TNF $\alpha$. Studies have shown that higher levels of adhesion molecules (i.e. E-Selectin, Intercellular adhesion molecule-1 ICAM-1, and P-Selectin) were expressed from the endothelial cells of adipose tissue in obese mice (Nakamura et al. 2014). Interestingly, leptin has been demonstrated to promote the expression of adhesion molecules in CD4+ $\mathrm{T}$ cells (like ICAM-1) (Fernandez-Riejoset al. 2010). Evidence of leptin receptors on monocytes and vascular tissue implies that the role of excessive leptin is pivotal in causing this chronic low-grade inflammatory state by the release of pro-inflammatory cytokines (Martin et al. 2008). Leptin was also seen to behave as a monocytic attractant (Fernandez-Riejoset al. 2010). Essentially, the adipocyte hyperplasia in obesity causes increased release of leptin, which stimulates the production of pro-inflammatory cytokines (from monocytes/macrophages), adhesion molecules, and increased oxidative stress - all of which contribute to endothelial damage. Hyperleptinemia-induced pro-inflammatory cytokine release assists in maintaining the inflammatory state that results in the endothelial damage, hypertension, and eventually atherosclerosis.

\section{Baroreflex sensitivity}

The baroreflex sensitivity (BRS) counters the effect of SNS induced increase blood pressure, hence BRS is inversely proportional to age, heart rate, and blood pressure. It is important to note that the vagus parasympathetic nerve mediates the function of baroreceptor reflex as a modulator of the heart rate. Additionally, there is inverse relation 
South American Journal of Medicine

Special Edition 2016

between BRS and triglyceride levels and fasting plasma glucose, both of which are relevant to obesity profiles (Skrapari et al. 2007). It was revealed that there was a nearly $50 \%$ reduction of BRS in obese women who were otherwise healthy. Though SNS activity is elevated in the skeletal muscles and kidneys among obese humans, the cardiac sympathetic activity is minimally affected due to the role of baroreflex inhibition (da Silva et al. 2009). Therefore, it is of importance to note that the increase in heart rate in obesity is a function of the reduction of the parasympathetic function of the heart. A report has suggested that during aging, endogenously produced leptin contributed to reduce the BRS (Arnold et al. 2014). The chief determining factor for reduced BRS was due to the dominant role of sympathetic activity over parasympathetic. Cardiac vagal dysfunction in obesity has been shown to contribute blunted BRS (Skrapari et al. 2007). Weight loss has a twofold effect in decrease of sympathetic activity and improvement of cardiac vagal activity, as a result enhancing the BRS (Voulgari et al. 2013).

\section{Leptin and cardiac hormones}

Though there is much room for research, a potential for future treatment methods has been seen in four cardiac hormones: vessel dilator (VD), long-acting natriuretic peptide (LANP), kaliuretic peptide, and atrial natriuretic peptide (ANP) — all of which have blood pressure lowering properties. The heart synthesizes these four hormones, which originate from the same gene. Study of hypertension in obese individuals has shown elevated concentrations of ANP, LANP, and VD. This increase in the cardiac hormones has been observed to revert to normal as the patient losses weight to reduce their high blood pressure. The way by which these cardiac hormones influence blood pressure has been thought to be via decreasing the hypothalamic concentrations of leptin (Lane \& Vesely 2013).

\section{Conclusions}

The means by which leptin resistance occurs and the pathways leptin stimulates to induce hypertension are not yet fully understood. However, there is some exciting progress being made in this field that may soon lead to new pharmacological strategies, which possibly not only reduce the immediate cardiovascular risks associated with obesity-induced hypertension, but could also provide safe and effective weight loss therapeutics for obese individuals. Recent studies regarding the role of pro-inflammatory mediators IKK- $\beta / \mathrm{NF}-\kappa \mathrm{B}$ and the effect of this pathway on the arcuate nucleus, obesity, hypertension, and leptin resistance clearly provide an area that warrants further study. Various studies on different gene knockout experimental animals and reports on several anti-inflammatory phenomena have shown significant protection against leptin resistance and obesity, even when fed a high fat diet. These approaches may prove to be promising pharmacological avenues to combat obesity-associated comorbidities.

\section{References}

[1]. Arnold, A. C., \& Diz, D. I. (2014). Endogenous leptin contributes to baroreflex suppression within the solitary tract nucleus of aged rats. American Journal of Physiology - Heart and Circulatory Physiology, 307(11), H1539-1546.

[2]. Banks, W. A. (2008). The blood-brain barrier as a cause of obesity. Current Pharmaceutical Design, 14(16), 16061614.

[3]. Beltowski, J. (2012). Leptin and the regulation of endothelial function in physiological and pathological conditions. Clinical and Experimental Pharmacology and Physiology, 39(2), 168-178.

[4]. da Silva, A. A., Carmo, J. D., Dubinion, J., \& Hall, J. E. (2009). The role of the sympathetic nervous system in obesity-related hypertension. Current Hypertension Reports, 11(3), 206-211. 


\section{South American Journal of Medicine Special Edition 2016}

[5]. da Silva, A. A., Do Carmo, J. M., \& Hall, J. E. (2013). Role of leptin and CNS melanocortins in obesity hypertension. Current Opinion in Nephrology and Hypertension, 22(2), 135-140.

[6]. Fernandez-Riejos, P., Najib, S., Santos-Alvarez, J., Martin-Romero, C., Perez-Perez, A., Gonzalez-Yanes, C., \& Sanchez-Margalet, V. (2010). Role of leptin in the activation of immune cells. Mediators of Inflammation, 2010, 568343.

[7]. Freeman, R. H., Go, O., Reams, G. P., Spear, R., Liu, K., \& Villarreal, D. (2014). Obesity hypertension: pathophysiological role of leptin in neuroendocrine dysregulation. American Journal of the Medical Sciences, 347(6), 485-489.

[8]. Frühbeck, G. (2006). Intracellular signalling pathways activated by leptin. Biochemical Journal, 393(Pt 1), 7-20.

[9]. Hall, J. E., do Carmo, J. M., da Silva, A. A., Wang, Z., \& Hall, M. E. (2015). Obesity-induced hypertension: interaction of neurohumoral and renal mechanisms. Circulation Research, 116(6), 991-1006.

[10]. Haynes, W. G. (2005). Role of leptin in obesity-related hypertension. Experimental Physiology, 90(5), 683-688.

[11]. Humphreys, M. H. (2011). The brain splits obesity and hypertension. Nature Medicine, 17(7), 782-783.

[12]. Kalil, G. Z., \& Haynes, W. G. (2012). Sympathetic nervous system in obesity-related hypertension: mechanisms and clinical implications. Hypertension Research, 35(1), 4-16.

[13]. Korda, M., Kubant, R., Patton, S., \& Malinski, T. (2008). Leptin-induced endothelial dysfunction in obesity. American Journal of Physiology - Heart and Circulatory Physiology, 295(4), H1514-1521.

[14]. Lane, M. L., \& Vesely, D. L. (2013). Reduction of leptin levels by four cardiac hormones: Implications for hypertension in obesity. Experimental and Therapeutic Medicine, 6(2), 611-615.

[15]. Mantzoros, C. S., Magkos, F., Brinkoetter, M., Sienkiewicz, E., Dardeno, T. A., Kim, S. Y., Hamnvik, O. R., Koniaris, A. (2011). Leptin in human physiology and pathophysiology. American Journal of Physiology Endocrinology and Metabolism, 301(4), E567-584.

[16]. Martin, S. S., Qasim, A., \& Reilly, M. P. (2008). Leptin resistance: a possible interface of inflammation and metabolism in obesity-related cardiovascular disease. Journal of the American College of Cardiology, 52(15), 12011210.

[17]. Mattu, H. S., \& Randeva, H. S. (2013). Role of adipokines in cardiovascular disease. Journal of Endocrinology, 216(1), T17-T36.

[18]. Nakamura, K., Fuster, J. J., \& Walsh, K. (2014). Adipokines: A link between obesity and cardiovascular disease. Journal of Cardiology, 63(4), 250-259.

[19]. Olofsson, L. E., Unger, E. K., Cheung, C. C., \& Ku, A. W. (2013). Modulation of AgRP-neuronal function by SOCS3 as an intiating event in diet-induced hypothalamic leptin resistance. Proceeding of the National Academy of Sciences, 110(8), E697-E706.

[20]. Purkayastha, S., Zhang, G., \& Cai, D. (2011). Uncoupling the mechanisms of obesity and hypertension by targeting hypothalamic IKK- $\beta$ and NF-אB. Nature Medicine, 17(7), 883-887.

[21]. Pedroso, J. A., Buonfiglio, D. C., Cardinali, L. I., Furigo, I. C., Ramos-Lobo, A. M., Tirapegui, J., Elias, C. F., Donato J. Jr. (2014). Inactivation of SOCS3 in leptin receptor-expressing cells protects mice from diet-induced insulin resistance but does not prevent obesity. Molecular Metabolism, 3(6), 608-618.

[22]. Ray, A., \& Cleary, M. P. (2010). Leptin as a potential therapeutic target for breast cancer prevention and treatment. Expert Opinion on Therapeutic Targets, 14(4), 443-451.

[23]. Reed, A. S., Unger, E. K., Olofsson, L. E., Piper, M. L., Myers, M. G., \& Xu, A. W. (2010). Functional role of suppressor of cytokine signaling 3 upregulation in hypothalamic leptin resistance and long-term energy homeostasis. Diabetes, 59(4), 894-906.

[24]. Rivest, S. (2002). Does circulating leptin have the ability to cross the blood-brain barrier and target neurons directly? Endocrinology, 143(9), 3211-3213.

[25]. Simonds, S. E., \& Cowley, M. A. (2013). Hypertension in obesity: is leptin the culprit? Trends in Neurosciences, 36(2), 121-132.

[26]. Skrapari, I., Tentolouris, N., Perrea, D., Bakoyiannis, C., Papazafiropoulou, A., Katsilambros, N., \& Ioanna, S. (2007). Baroreflex sensitivity in obesity: relationship with cardiac autonomic nervous system activity. Obesity (Silver Spring), 15(7), 1685-1693. 


\section{South American Journal of Medicine Special Edition 2016}

[27]. Voulgari, C., Pagoni, S., Vinik, A., \& Poirier, P. (2013). Exercise improves cardiac autonomic unction in obesity and diabetes. Metabolism, 62(5), 609-621.

[28]. Wang, J., Wang, H., Luo, W., Guo, C., Wang, J., Chen, Y. E., \& Chang, L. (2013). Leptin-induced endothelial dysfunction is mediated by sympathetic nervous system activity. Journal of American Heart Association, 2(5), e000299.

[29]. Yang, R., \& Barouch, L. A. (2007). Leptin signaling and obesity: Cardiovascular consequences. Circulation Research, 101(6), 545-559.

[30]. Zhang, X., Zhang, G., Zhang, H., Karin, M., Bai, H., \& Cai, D. (2008). Hypothalamic IKKb/Nf-kB and ER stress link overnutrition to energy imbalance and obesity. Cell, 135(1), 61-73.

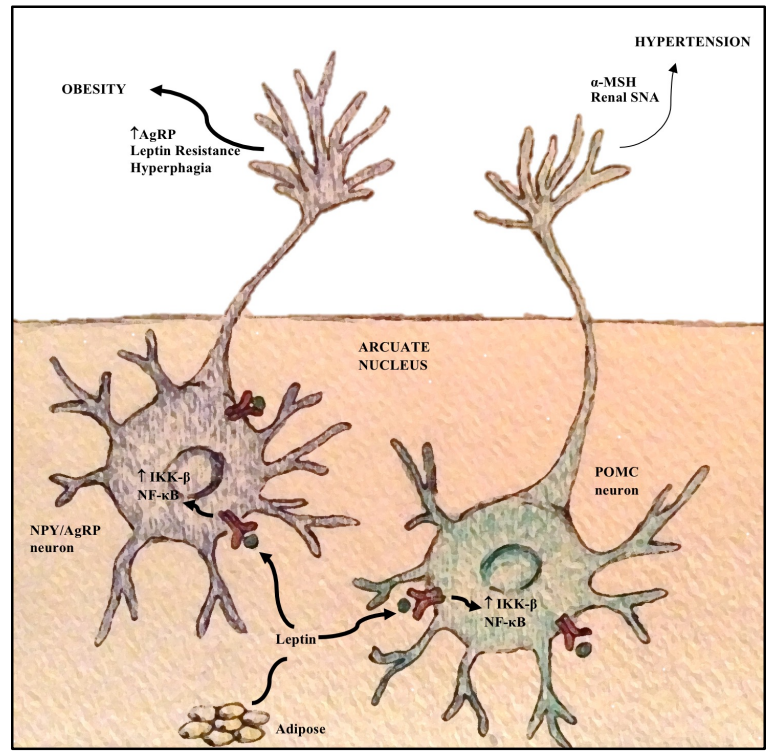

Figure 1. Physiological functions of leptin. Leptin maintains the balance between food intake and energy expenditure. Leptin works on many body functions, conserving energy during times of food scarcity and expending energy during times of abundance. SNA: Sympathetic nerve activity. 


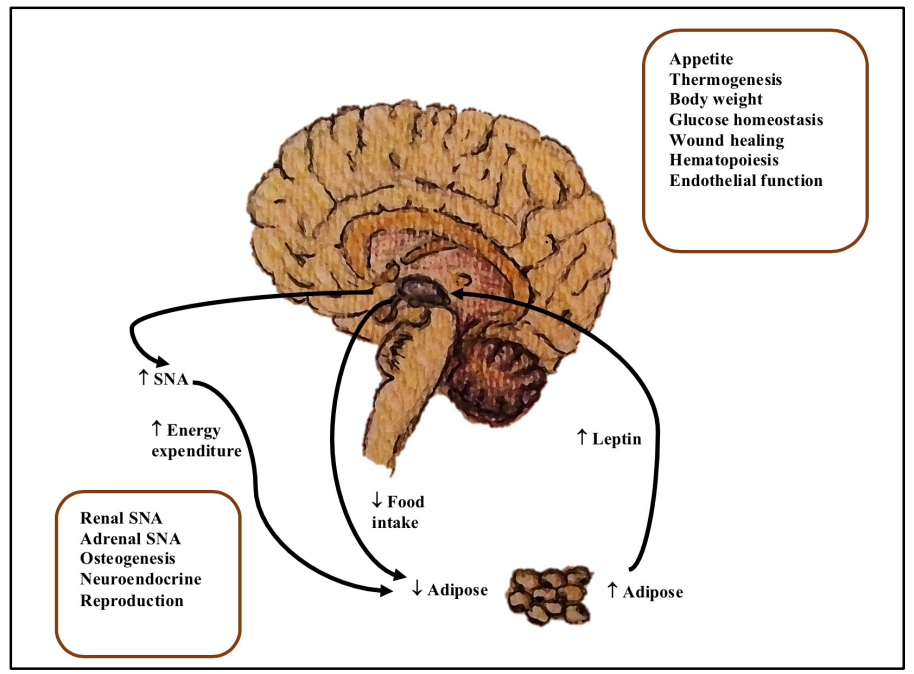

Figure 2. IKK $-\beta / N F-\kappa B$ axis is stimulated in both the POMC and NPY/AgRP neurons of the arcuate nucleus. In the POMC neurons, IKK- $\beta / N F-\kappa B$ promotes the melanocortin system, leading to renal sympathetic nerve stimulation and hypertension. In the NPY/AgRP, IKK- $\beta / N F-\kappa B$ leads to increased AgRP production, leptin resistance and hyperphagia.



Figure 3. Leptin uses a complex system of intracellular pathways to perform its homeostatic role. Tyrosine 1138 on $\mathrm{Ob}-\mathrm{Rb}$ is essential for STAT3 phosphorylation and the stimulation of SOCS3. Along with SOCS3, PTP1B and SHP2 are also capable of inhibiting leptin signaling. Leptin binding has different downstream effects, depending on the cell type. This allows for selective leptin resistance in the obese, chronic hyperleptinemic state.

\section{Acknowledgement}

We are grateful to Professor Claude-Bernard lliou, M.D., Dean, Saint James School of Medicine - Anguilla, for his constant support. Moreover, we would like to thank our Librarian Oscar Andrade, M.S.,M.L.I.S., for necessary assistance. 